\title{
Approaches to Low Fuel Regression Rate in Hybrid Rocket Engines
}

\author{
Dario Pastrone \\ Dipartimento di Ingegneria Meccanica e Aerospaziale, Politecnico di Torino, Corso Duca degli Abruzzi 24, 10129 Torino, Italy \\ Correspondence should be addressed to Dario Pastrone, dario.pastrone@polito.it \\ Received 10 February 2012; Accepted 5 April 2012 \\ Academic Editor: David Greatrix \\ Copyright () 2012 Dario Pastrone. This is an open access article distributed under the Creative Commons Attribution License, \\ which permits unrestricted use, distribution, and reproduction in any medium, provided the original work is properly cited. \\ Hybrid rocket engines are promising propulsion systems which present appealing features such as safety, low cost, and \\ environmental friendliness. On the other hand, certain issues hamper the development hoped for. The present paper discusses \\ approaches addressing improvements to one of the most important among these issues: low fuel regression rate. To highlight the \\ consequence of such an issue and to better understand the concepts proposed, fundamentals are summarized. Two approaches \\ are presented (multiport grain and high mixture ratio) which aim at reducing negative effects without enhancing regression rate. \\ Furthermore, fuel material changes and nonconventional geometries of grain and/or injector are presented as methods to increase \\ fuel regression rate. Although most of these approaches are still at the laboratory or concept scale, many of them are promising.
}

\section{Introduction}

Hybrid rocket engines (HREs) are chemical rockets which present interesting advantages over liquid rocket engines (LREs) and solid rocket motors (SRMs) and can provide a safe and affordable option for many applications. They have recently come to the fore as they have been chosen to power the second stage of the Tier One, the winner of the $\$ 10$ million X-Prize [1]. The Tier One (http://www.scaled.com/projects/tierone/) was the first privately-developed reusable vehicle able to perform a manned suborbital flight over $100 \mathrm{~km}$. The first stage of the Tier One is a twin-turbojet carrier-launch aircraft, called the White Knight, which is able to bring the second stage to $15 \mathrm{~km}$ altitude. The second stage, the SpaceShipOne, is a reusable three-place manned space plane, powered by an $\mathrm{N}_{2} \mathrm{O} / \mathrm{HTPB}$ hybrid rocket engine. This HRE has a burning time of about 80 seconds and it is able to produce an average thrust of $75 \mathrm{kN}$ with a vacuum specific impulse of about 250 s. The Tier One was retired after winning the X-Prize, but it remains proof that HREs may be the key propulsion system of the emerging space market [2].

In HREs, oxidizer and fuel are separated and stored in two different physical phases. In the most common configuration, a liquid or gaseous oxidizer and a solid-fuel grain are employed (direct HRE). In the conventional configuration the grain is cylindrical with a circular port. Due to this propellant storage, HREs have a peculiar combustion process as shown Figure 1. A boundary layer is formed when the oxidizer is injected into the port of the fuel grain. After ignition a diffusion flame is formed inside this layer. The fuel deriving from the solid grain is gasified by the heat coming from the flame, which in turn is fed by the fuel. The gasified fuel blows from the surface and modifies the boundary layer, blocking the heat transfer.

The aforementioned propellant storage and combustion process determine both positive and negative effects. Many authors have discussed the advantages and the drawbacks of HREs. Characteristic hybrid rocket features are briefly summarized below. Benefits include the following.

(i) Performance: HREs may deliver a higher specific impulse than SRMs. Due to the high-density solid they also may have higher density specific impulse than LREs. This latter benefit may be wasted by sliver or low grain volumetric efficiency.

(ii) Safety: HREs are inherently safe and low cost mainly due to the use of a solid fuel grain which is classically inert. Since the fuel and oxidizer are separated by distance and phase, hybrids have almost no explosion 


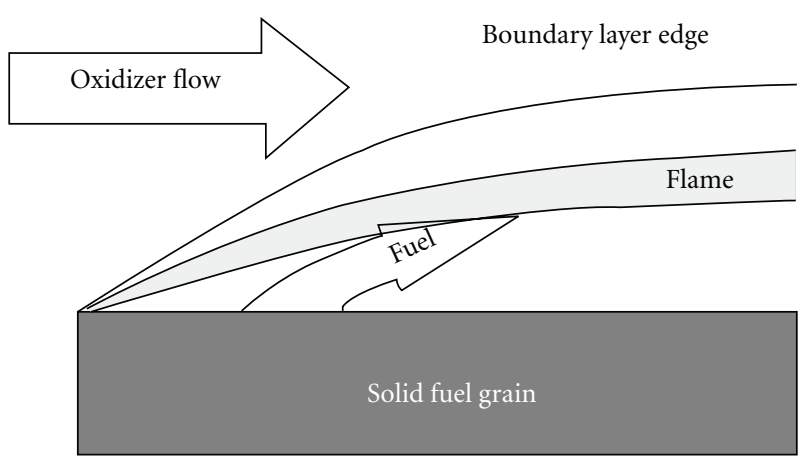

FIgURE 1: HRE combustion.

hazard and very few failure modes. The fluid oxidizer is usually depleted in the diffusion flame region. It can reach and attack the fuel surface only when gaseous chemical kinetics are slow (low pressures). No deflagration to detonation transition can be caused by pores, cracks, and imperfections, and inadvertent ignitions are avoided. Last, but not least, defining a maximum expected operating pressure is not a concern as HREs do not have the high temperature sensitivity which characterizes SRMs.

(iii) Reliability and simplicity: feeding system hardware is reduced as only the oxidizer is liquid.

(iv) Fuel versatility: additives for different purposes can be embedded in the fuel grain.

(v) Oxidizer control: liquid propellant control allows for throttling, motor shutdown, and reignition.

(vi) Environmental friendliness: compared to SRMs, oxidizers are chlorine free. Compared to LREs, storable propellants are available, which do not have noxious features such as $\mathrm{MMH}$ and nitrogen tetroxide.

(vii) Low cost: hybrid rockets pose almost no explosion hazard during manufacture, transport, ground test, and storage. Low recurring costs are foreseen because of high levels of safety and minimal failure modes.

Classical HREs also present disadvantages which include the following.

(i) Performance: HREs cannot reach the high specific impulse of cryogenic bipropellant LREs and have a lower density specific impulse compared to SRMs.

(ii) Low fuel regression rate: the regression rate of conventional binders such as HTPB is typically an order of magnitude lower than solid propellants and hence a large fuel surface is needed to produce the required thrust level, as discussed later in Section 2. This limit is usually set by the physical phenomenon of heat transfer from the diffusion flame to the fuel surface. As a consequence, HREs may have poor fuel loading, low thrust densities, and large length-todiameter ratios.

(iii) Mixture ratio shifting: the regression rate primarily depends on the mass-flux, that is, on the ratio of mass-flow and port area $A_{p}$. During combustion of classical side burning grains, $A_{p}$ increases determining a regression rate reduction. On the other hand, the burning area $A_{b}$ increases as well. These two competing effects are, in general, not balanced, leading to a change in the fuel mass flow, even when the oxidizer mass-flow is kept constant (see Section 2). The mixture ratio shifts and performance are worsened. Nonconventional injection systems may fix this issue but introduce complexity.

(iv) Mixing inefficiencies: part of the fuel under the flame at the grain port exit may not mix with any oxidizer and thus exit the nozzle before releasing chemical energy. HREs have a lower overall combustion efficiency than LREs and SRMs. A mixer between the grain aft end and the nozzle inlet can enhance mixing, but the dry weight of the system is larger.

(v) Slow transient/response to throttling: due to the thermal lag in the solid fuel, ignition and response to throttling is slow in comparison to LREs. Also, the chamber volume may be larger than in LREs, with large tail-off time.

One of the most important issues remains the very low regression rate of the fuel grain. Various methods for enhancing regression rate or, at least, for reducing negative effects of low fuel regression have been suggested in the past. The goal of this paper is to survey broadly some of the more significant approaches proposed. After summarizing some fundamentals (Section 2), two approaches are presented (Section 3) which aim at reducing the negative effects of regression rate without enhancing regression rate itself. Approaches which directly try to enhance regression rate are then presented: the modification of fuel is considered in Section 4, while approaches based on unconventional geometries for the grain and/or the injector are presented in Section 5 .

\section{Fundamentals}

In this section the fundamentals are summarized in order to better understand the negative effects of low regression rate and in turn to allow one to consider approaches to mitigate this issue. An exhaustive treatment of this subject is beyond the scope of this work. Rudiments of regression rates behavior and models are first discussed. A ballistic model is then used to highlight that cylindrical single-port sideburning grain may have an unacceptable shape if large thrust levels are required. For further information consult [14].

2.1. Regression Rate Behavior and Modeling. Typical fuel regression rate behavior is depicted in Figure 2 as a function of mass flux $G$. Three different regions can be identified. For the medium $G$ range the regression rate is diffusion dominated and is a function of the mass flux [15-17]. When $G$ assumes high values, the combustion appears to be controlled by chemical kinetics and not by diffusion [18]. As a consequence, regression rate becomes pressure 


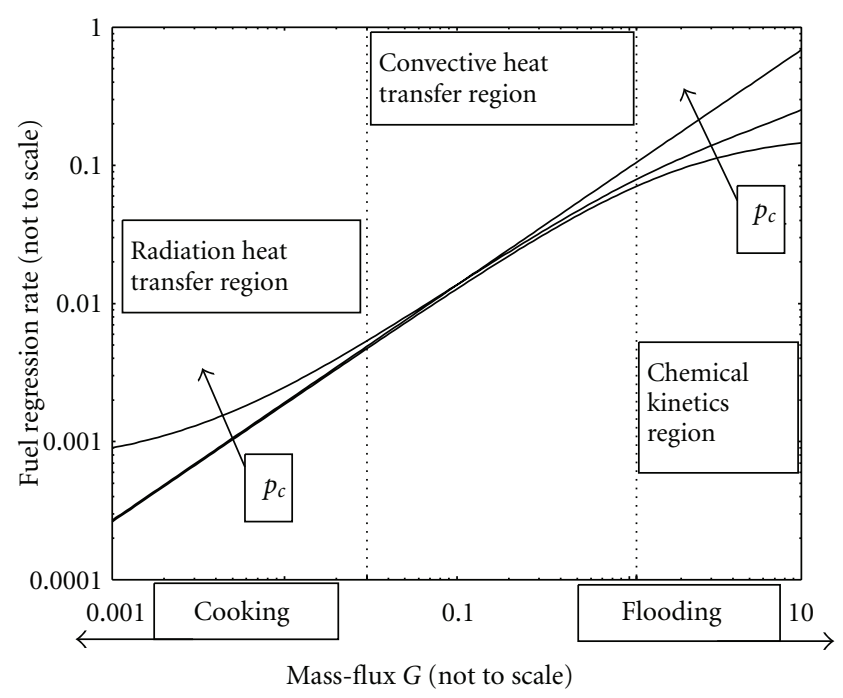

FIgURE 2: Typical regression rate behavior.

dependent. An upper bound for the mass flux exists, called the flooding limit, which depends on pressure level and propellant combination. When approaching this limit, the mass flux may become so high that the flame is extinguished due to small Damköhler numbers and/or very oxidizer-rich conditions. Finally, at low values of mass flux, the convective heat transfer diminishes and radiation from gaseous species may play a significant role. Consequently, the regression rate is enhanced depending on the partial pressure of emitting components and port diameter. In this regime a lower bound of the mass flux is also present. The regression rate is small, the fuel remains in the thermal layer of the grain a long time, and the solid fuel may be cooked/melt underneath the grain surface. Therefore, if the mass flux is too low (e.g., at the end of HRE operation with blowdown feed systems), chuffing instability may be produced by the perpetual repeating mechanical removal of this soft layer.

The well-known theory developed by Marxman and colleagues [15-17] set the basis for understanding and describing fuel regression rate. They assumed that the fuel regression rate is controlled by heat transfer to the grain and applied a flux balance at the fuel surface, finding

$$
r \rho_{F}=\frac{q}{\Delta H_{v, \mathrm{eff}}}
$$

where $r$ is the fuel regression rate, $\rho_{F}$ is the fuel density, $q$ is the total heat flux received by the fuel grain, and $\Delta H_{v \text {,eff }}$ is the thermal energy required to change a unit mass of solid fuel into gas.

According to their studies, the regression rate is primarily governed by convective heat transfer, that is, by the local mass flux $G$. Modeling the heat transfer in a turbulent layer, they proposed a simplified regression rate expression for combustion with negligible radiation. Taking into account a refit of the data proposed by Altman Humble [19] this expression is

$$
r \rho_{F} \propto B^{0.32} G^{0.8} x^{-0.2}, \quad 5<B<100 .
$$

The blowing parameter $B$ is the ratio of the thermal energy of the main stream relative to the surface $\Delta H_{f w}$ and $\Delta H_{v \text {,eff }}$ The weak negative dependence on axial position $x$ reflects the effects of boundary layer growth on heat transfer. On the contrary, the total local mass flux increases with axial position along the fuel grain. These two competing effects usually determine a location of minimum regression rate along the grain axis. In any case, these difference are usually small. For these reasons the regression rate is assumed to be constant along the grain axis and semiempirical correlations based on the mass flow entering the port area, that is, $G_{O}=$ $\dot{m}_{O} / A_{p}$ are widely used, that is,

$$
r=a G_{O}^{n}
$$

where $a$ and $n$ are obtained by experimental data for a given $G_{O}$ range and depend on engine dimensions (scale effect), port/grain geometry, injector geometry, and flow features. Correlation like the one presented in (3) well-describe the regression rate of conventional HREs in the intermediate range of $G_{O}$, where behavior is dominated by turbulent heat transfer.

Radiation from gas-phase products is usually small compared to convection. Nevertheless, convection itself becomes small at low values of $G$ and radiation plays a role. Radiation effects are also important when the combustion products contain condensed matter, for example, soot and metal/oxides particles. Marxman and coworkers [16, 17] proposed a correction factor to take into account the radiation heat transfer. The total heat flux $q$ in (1) becomes $q=q_{c}\left[\left(q_{r} / q_{c}\right)+\exp \left(-q_{r} / q_{c}\right)\right]$. This expression takes into account that there is a coupling effect between radiant flux $q_{r}$ and convective heat $q_{c}$. In fact, the radiant flux enhances the blocking effect due to blowing thus depressing convective heat.

Other phenomena that affect fuel regression rate have been considered in later works [20-28]. Beside radiation, the most important mechanisms taken into account include variable fluid and transport properties across the boundary layer, and both gas-phase and heterogenous reaction kinetics.

The regression rate correlation of (3) should be modified consequently. As an example, Chiaverini et al. [28] proposed a modified relation for cases where kinetics effects can be disregarded. Variable fluid and transport properties into the boundary layer and radiation from soot are considered. The resulting model is

$$
\frac{\rho_{F} r}{G}=0.0155\left(\frac{D_{h}}{L_{g}}\right)^{0.3} \theta^{0.6} \operatorname{Re}_{D}^{-0.2} f\left[B, \frac{q_{r}}{q_{c}}\right],
$$

where $D_{h}$ is the port hydraulic diameter, $L_{g}$ the grain length, and $\theta$ a temperature factor, representing the ratio of average flame to surface temperature [29].

Although correlations like (4) allow for a better fit of experimental data, (3) is often used. The reader should be aware that the values of $a$ and $n$ may be dependent on many factors such as $G_{O}$ range, fuel formulation, grain production method, engine dimensions (scale effect), port/grain geometry, injector geometry, and flow features. 
For example, if (3) is used to fit experimental data, $r$ may be found to vary with $G_{O}$ to the power of 0.6 instead of the theoretical 0.8 for turbulent flow over a flat plate used in (2). These findings imply that nonconvective processes, such as radiant heat flux and/or finite rate chemical kinetics, may influence the solid-fuel regression rates in this case.

\subsection{Low Fuel Regression Rate Effects in Classical HREs.} When the propellant combination is given, the characteristic velocity $c^{*}$ is a function of the mixture ratio $\alpha=\dot{m}_{O} / \dot{m}_{F}$ (i.e., oxidizer to fuel mass flow ratio) [3]. Chamber pressure affects $c^{*}$ to a lesser extent. The thrust coefficient $C_{F}$ can be evaluated when the following data are provided: combustion gas properties (specific heat ratio), ambient pressure $p_{a}$, and expansion ratio $\epsilon=A_{e} / A_{t}$ (exit to throat area ratio) or $p_{e} / p_{c}$ (exit to chamber pressure ratio). The corresponding effective exhaust velocity $c=c^{*} C_{F}$ or the specific impulse $I_{s}=c / g_{0}$ is consequently evaluated.

If a thrust level is assigned, the needed propellant mass flow $m_{p}$ is

$$
\dot{m}_{p}=(1+\alpha) \dot{m}_{F}=\frac{F}{c^{*} C_{F}},
$$

and, using (3), the fuel mass flow $m_{F}$ is

$$
\dot{m}_{F}=a \rho_{F}\left(\frac{\dot{m}_{O}}{A_{p}}\right)^{n} A_{b}
$$

where $\rho_{F}$ is the solid fuel density.

The mixture ratio is

$$
\alpha=\frac{\dot{m}_{O}}{\dot{m}_{F}}=\frac{\dot{m}_{O}^{(1-n)}}{a \rho_{F}} \frac{A_{p}^{n}}{A_{b}} .
$$

This relation shows that $\alpha$ changes in proportion to $A_{b} / A_{p}^{n}$. In most cases $A_{b} / A_{p}^{n}$ is a function of time and $\alpha$ changes even if $\dot{m}_{O}$ is kept constant. This characteristic behavior of HREs is called mixture-ratio shifting. It is worthwhile to observe that mixture ratio shifting does not occur if a circular port is used and $n=0.5$.

From (5) and (6):

$$
A_{b}=\frac{1}{a \rho_{F}}\left(\frac{F}{c^{*} C_{F}}\right)^{(1-n)} \frac{A_{p}^{n}}{(1+\alpha)^{(1-n)} \alpha^{n}} .
$$

Equation (8) shows the relation of geometry (grain/nozzle) engine operation (mixture ratio) and mission requirements (thrust). Due to the low regression rate, the length-todiameter ratio may be of concern. In fact, large values of $A_{b}$ are required, while $A_{p}$ must keep $G_{O}$ in the appropriate range. Assuming a single-circular port, for given values of $F$, $\alpha$, and $c$, the length-to-diameter ratio of the grain becomes $\left(L_{g} / D_{p}\right) \propto D_{p}^{(2 n-1)}$, where $L_{g}$ is the grain length, and $D_{p}$ the port diameter. The outer diameter of the grain is $D_{g}=$ $D_{p}+2 w$, where $w$ is the web thickness. If $n>0.5$ the lengthto-diameter ratio of the grain may be reduced with a port diameter reduction. On the other hand, the overall lengthto-diameter ratio $L / D$ must be considered. The rocket length $L$ includes the oxidizer tank length. The reduction of the port area makes the ratio $L / D$ worse if the oxidizer tank and grain have the same diameter [30]. On the contrary, if the diameter of the oxidizer tank may be larger that grain diameter, both $L$ is reduced and $D$ augmented thus improving the ratio $L / D$. Therefore, the reduction of $A_{p}$ may be favorable [31]. However, this approach is limited as $A_{p}$ has a lower bound due to flooding.

As a consequence, HREs are generally restrained to high length-to-diameter ratios and thus are long and skinny. The values of $L / D$ become unacceptable when requiring high performance (e.g., upper stages and boosters), especially when compared to LREs and SRMs that are currently on the market. Solutions to this issue become necessary.

\section{Mitigation Approaches}

In this section two solutions which attempt to mitigate the negative effects of low regression rate are presented. Multiport grains allow for reducing $L / D$ values with an almost constant fuel mass, while high mixture ratio aims at reducing the mass of the fuel grain.

3.1. Multiport Grains. Assuming that both $A_{b}$ and $A_{p}$ are constant, the grain length $L_{g}=A_{b} / P$ may be reduced by increasing the perimeter $P$. This result can be obtained using a multiport grain. The larger the number of ports, the more pronounced the length reduction. Also, volumetric efficiency is increased as the port volume is proportional to $L_{g}$. As an example, two possible multiport geometries are shown in Figure 5. In Figure 5(a) a triangular port [32] is shown. Sliver is a drawback which can be reduced using a wagon-wheel grain (Figure 5(b)) where a central circular port with initial radius $R_{c i}$ is surrounded by a row of $N$ quadrangular ports [33]. Grains with two or three rows of quadrangular ports have been tested [30]. Even with these more complicated geometries the unburned mass fraction is high. Furthermore, other drawbacks exist. The individual ports may behave in a different way and dedicated injector or a large prechamber would be required, compromising weight and simplicity. In addition, design and fabrication are complex and grain structural integrity may become an issue, especially towards the end of the burn.

Radial-flow/end-burning grains are other interesting geometry alternatives which may improve fuel section volumetric efficiency. Combination of end-burning with sideburning grains is possible. In this case the flow structure is modified and the regression rate may be enhanced too. Approaches which involve end burning grain or radial flows are left for the next sections which discuss regression rate enhancement.

Also more complicated geometries can be used which allow for a better use of volume. Rapid prototyping has been identified as a manufacturing technique for hybrid rocket fuel grains that enables the production of complex $3 \mathrm{D}$ grain shapes, which may improve hybrid rocket motor performance [34].

3.2. High Mixture-Ratio Values. If high mixture-ratio values are used, the regression rate is of minor concern, as the fuel 


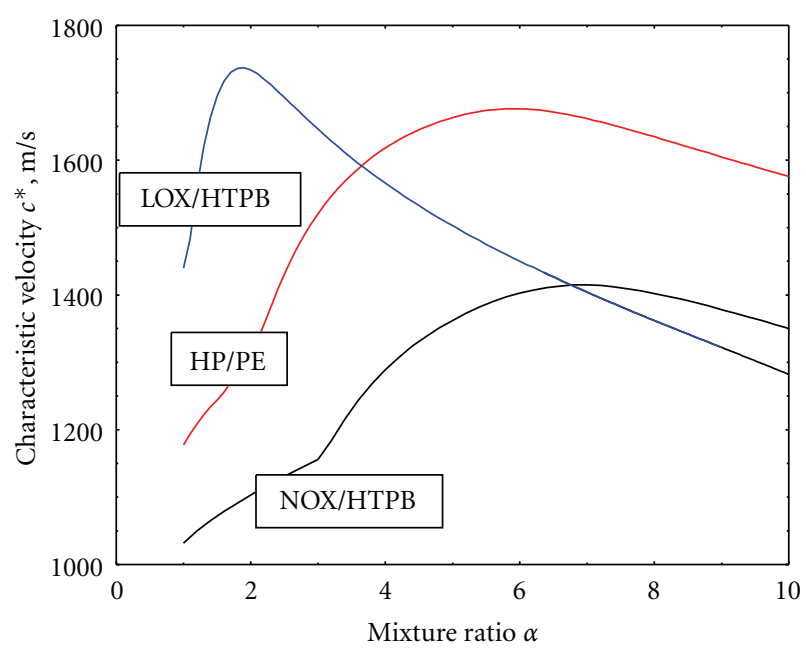

FIGURE 3: Theoretical values of characteristic velocity for different propellant combinationsm [3].

grain must give a minor contribution to the overall mass flow. Unfortunately, the propellant performance is a function of mixture ratio as well. Benefits may be obtained by adopting propellant combinations which present high values of $c^{*}$ at high mixture rations. Figure 3 shows $c^{*}$ for three different propellant combinations: HTPB with liquid oxygen (LOX) or $\mathrm{N}_{2} \mathrm{O}$, and polyethylene (PE) with an $85 \%$ concentration solution of hydrogen peroxide (HP). The thrust coefficient $C_{F}$ is primarily a function of the expansion ratio and is only slightly affected by $\alpha$, reaching a maximum value in the stoichiometric region. As a result the maximum value of $c$ is somehow shifted to the right of $\alpha$ which determines the maximum of $c^{*}$.

It is worthwhile to note here that proper tools are needed to perform a comparison [30, 31, 35-37]. In many applications thrust has a major influence since it affects both the propulsion system design and the trajectory performance; a compromise must be sought, as greater thrust levels reduce the gravitational losses but increase structural mass. Moreover, HREs are characterized by their peculiar combustion process and the consequent link between thrust level and mixture ratio. In order to efficiently perform a coupling of the HRE design parameter optimization with the trajectory optimization, a unique procedure has been developed at the Politecnico di Torino which uses a direct/indirect nested method.

As an example, the performance of a microgravity platform for three different propellant combination are compared [31]. Given rocket payload $(100 \mathrm{~kg})$ and initial mass (500 kg, comprising payload, fixed masses, propulsion system, and propellant), the time spent above $100 \mathrm{~km}\left(t_{\mu g}\right)$ is the performance index to be maximized. The optimization aims at finding the optimal mass split between propellant and propulsion system (i.e., tanks, combustion chamber, nozzle), the optimal grain geometry, and the corresponding optimal trajectory [31]. Single-port grains are considered and different propellant options are compared in Table 2. A simple blowdown feed system is adopted.

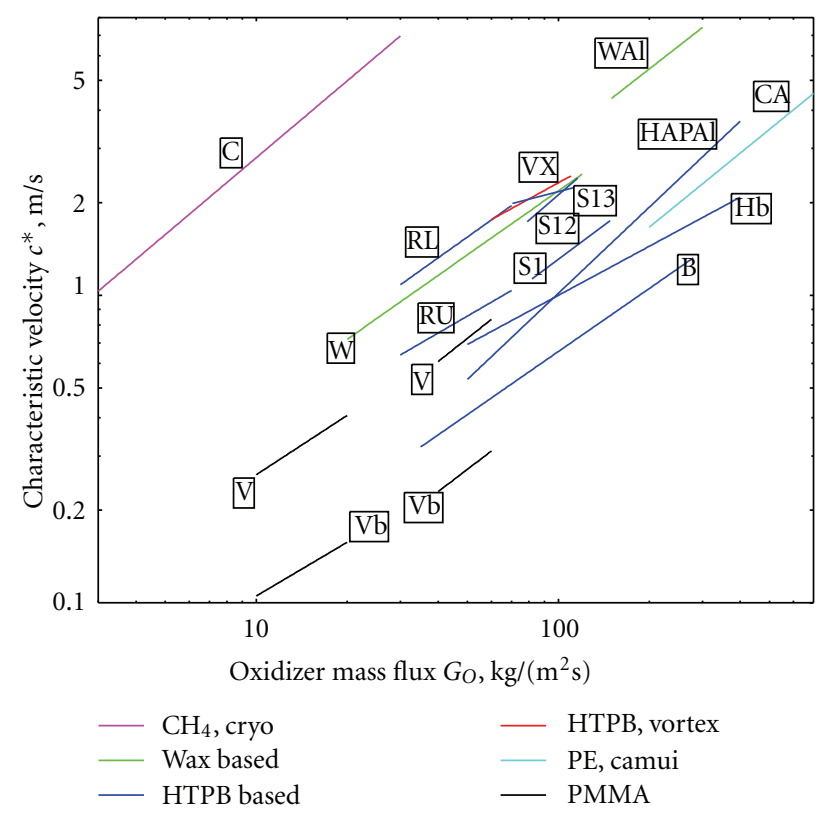

Figure 4: Regression rate for different systems and GOX/fuel combinations (see legend in Table 1).

Results show the superior performance of the HP/PE combination. Propellant consumption and propulsion system dry-mass are competing parameters. The LOX/HTPB propellant combination presents higher maximum values of $c^{*}$, but this maximum is reached for small values of $\alpha$ : large grains are required and the propulsion system mass penalty is higher than propellant savings. Also, LOX and HP have larger densities than HTPB, and higher values of density-specific impulse may be obtained with the HP/PE combination which adopts high values of $\alpha$. On the other hand, $\mathrm{N}_{2} \mathrm{O}$ is penalized by the low performance in terms of specific impulse. However, $\mathrm{N}_{2} \mathrm{O}$ presents some interesting features, such as the reduced rocket length $L$ and diameter $D$, as the large mixture ratio reduces the grain length. Also note that the initial required thrust for the same mission is lower.

\section{Fuels with Enhanced Regression Rate}

In this section approaches are considered based on the modification of the fuel. Possible approaches can be divided down into three categories: (1) adding energetic particles, (2) using energetic polymers (and/or plasticisers) instead of conventional inert components such as HTPB, (3) using fuels such as cryogenic fuels or paraffin-based fuels which exhibit new mass-transfer mechanisms. A combination of the aforementioned options is also possible.

4.1. Particle Additives in Solid Fuels. Several additives have been tested. Most researches considered metal additives in polymeric-based solid fuels [6, 8, 9, 27, 41-45]. More recently, metal additives in paraffin-based fuels have also been considered $[8,46-50]$.

Combustion of metals has inherent advantages as they help to increase both specific impulse and density specific 
TABLE 1: Values of $a$ and $n$ to be used in (3) ( $r$ in m/s and $G_{O}$ in $\left.\mathrm{kg} / \mathrm{m}^{2} \mathrm{~s}\right)$.

\begin{tabular}{lccccccc}
\hline Legend & System & Propellants & $a\left(\mathrm{~m}^{1+2 n} \mathrm{~kg}^{-n} \mathrm{~s}^{n-1}\right)$ & $n$ & Note & Reference & $G_{O}\left(\mathrm{~kg} / \mathrm{m}^{2} / \mathrm{s}\right)$ \\
\hline & Fuel/additives & & & & & & \\
B & Pure HTPB & GOX/HTPB & $2.85 \cdot 10^{-5}$ & 0.681 & - & {$[4]$} & $35-280$ \\
W & Paraffin & GOX/Wax & $9.10 \cdot 10^{-5}$ & 0.690 & - & {$[5]$} & $20-120$ \\
WAl & Paraffin/13\% Silbal & GOX/fuel & $9.40 \cdot 10^{-5}$ & 0.766 & - & {$[6]$} & $150-300$ \\
C & Cryo & GOX/CH4 & $4.14 \cdot 10^{-5}$ & 0.830 & - & {$[7]$} & $3-30$ \\
S1 & Pure HTPB & GOX/fuel & - & - & baseline, [8, Figure 11] & {$[8]$} & $80-150$ \\
S12 & HTPB/13\% Al325 & GOX/fuel & - & - & {$[8$, Figure 11] } & {$[8]$} & $80-120$ \\
S13 & HTPB/13\% ALEX & GOX/fuel & - & - & coated, [8, Figure 11] & {$[8]$} & $70-120$ \\
Hb & Pure HTPB & GOX/HTPB & $8.7 \cdot 10^{-5}$ & 0.530 & baseline & {$[9]$} & $50-400$ \\
HAl & HTPB/Al & GOX/fuel & $1.4 \cdot 10^{-5}$ & 0.930 & - & {$[9]$} & $50-400$ \\
HAP & HTPB/AP & GOX/fuel & $3.8 \cdot 10^{-5}$ & 0.710 & - & {$[9]$} & $50-400$ \\
HAPAl & HTPB/Al/AP & GOX/fuel & $1.2 \cdot 10^{-5}$ & 0.97 & - & {$[9]$} & $50-400$ \\
\hline & Swirl/grain geometry & & & & & & \\
VX & Vortex & GOX/HTPB & $1.93 \cdot 10^{-4}$ & 0.540 & - & {$[10]$} & $60-110$ \\
V & End-burning + Swirl & GOX/PMMA & $3.45 \cdot 10^{-5}$ & 0.778 & - & {$[11]$} & $40-60$ \\
Vb & End-burning + Swirl & GOX/PMMA & $1.45 \cdot 10^{-5}$ & 0.749 & no swirl & {$[11]$} & $40-60$ \\
V & End-burning + Swirl & GOX/PMMA & $5.96 \cdot 10^{-5}$ & 0.641 & - & {$[11]$} & $10-20$ \\
Vb & End-burning + Swirl & GOX/PMMA & $2.76 \cdot 10^{-5}$ & 0.581 & no swirl & {$[11]$} & $10-20$ \\
RU & Radial & GOX/HTPB & $9.20 \cdot 10^{-5}$ & 0.570 & upper disk & {$[12]$} & $30-70$ \\
RL & Radial & GOX/HTPB & $1.00 \cdot 10^{-4}$ & 0.700 & lower disk & {$[12]$} & $30-70$ \\
CA & CAMUI & GOX/PE & $2.40 \cdot 10^{-5}$ & 0.800 & $(11), \alpha^{\prime}=3$ & {$[13]$} & $200-700$ \\
\hline
\end{tabular}

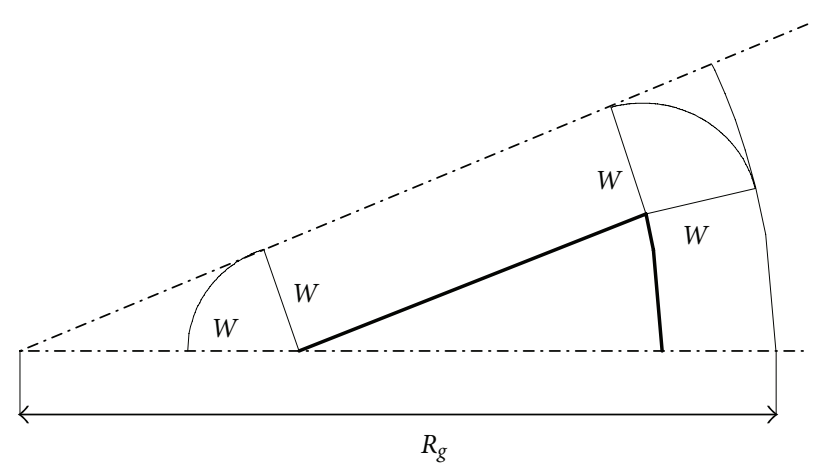

(a) Triangular-port geometry

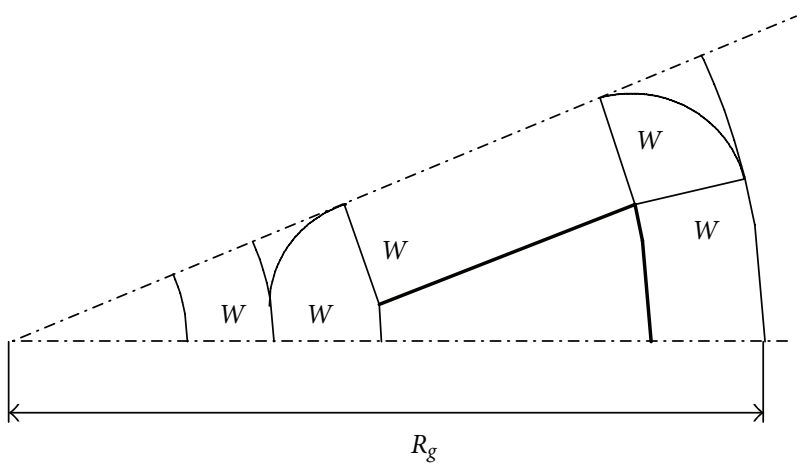

(b) Quadrilateral-port geometry

FIGURE 5: Multiport geometries.

impulse. In fact, even if their condensed combustion products determine multiphase losses, they have high reaction heat and high density. Being usually in powdered form they can easily be mixed in solid fuels. In the case of HREs it has been proven that metal additives may enhance regression rate. The addition of various types of metals (e.g., $\mathrm{Al}, \mathrm{LiH}, \mathrm{B}$, $\mathrm{W}, \mathrm{Mg}$ ) into solid fuels has been tested. Most investigations considered aluminum, lithium, and boron.

Regression rate is influenced by component characteristics (e.g., reactivity, heat of oxidation, density) and particle features (e.g., shape, dimension, coating). Aluminum may be considered the most researched additive. It has a high heat of oxidation, high density and ease of ignition. Lithiumbased particles are highly reactive, but the heat of oxidation is low compared to more common particles such aluminum. Finally, boron has a very high heat of combustion but is not so easy to ignite, which is of concern in limited volume propulsion systems. As far as dimension is concerned, microsized particles have been extensively tested. More recently nanosized particles have been used. Nanosized particles have a very high surface to volume ratio. Compared to microsized particles, nanosized particles present reduced ignition and burning time, present a more complete combustion (high $c^{*}$ efficiency), and determine a larger increment od regression rate, but they are more expensive and complicate the grain production process.

Nonvolatile fuel particles (e.g., aluminum) in the grain have several effects, influencing both physical properties 
TABLE 2: Optimal design and performance of sounding rocket for different propellant combinations.

\begin{tabular}{|c|c|c|c|c|c|c|c|c|c|}
\hline Propellants & $a\left(\mathrm{~m}^{1+2 n} \mathrm{~kg}^{-n} \mathrm{~s}^{n-1}\right)$ & $n$ & Reference & $F_{i}(\mathrm{kN})$ & $\alpha_{i}$ & $m_{p}(\mathrm{~kg})$ & $D(\mathrm{~m})$ & $L(\mathrm{~m})$ & $t_{\mu g}(\mathrm{~s})$ \\
\hline $\mathrm{HP} / \mathrm{PE}$ & $7.00 \cdot 10^{-6}$ & 0.800 & {$[38]$} & 24.8 & 8.57 & 339 & 0.42 & 5.01 & 299 \\
\hline LOX/HTPB & $9.24 \cdot 10^{-6}$ & 0.852 & [39] & 23.5 & 3.20 & 328 & 0.46 & 5.52 & 219 \\
\hline $\mathrm{N}_{2} \mathrm{O} / \mathrm{HTPB}$ & $1.87 \cdot 10^{-4}$ & 0.347 & {$[40]$} & 16.2 & 10.92 & 340 & 0.40 & 4.75 & 177 \\
\hline
\end{tabular}

of the solid and the combustion process. Reasons behind some test results are currently under investigation. The main known effects due to nonvolatile particles are as follows.

(i) Particles such as metals have higher density than fuel binders, and the grain mean density changes with the percentage of weight added $k$. It is useful to consider both the regression rate $r$ and the mass burning rate $\rho_{F} r$ improvement. For example, if a weight fraction of $20 \%$ of ultrafine aluminum (UFAL) is added in $\mathrm{HTPB}$, the regression rate is increased by about $40 \%$, whereas the mass burning rate increases by almost $70 \%$ over that of pure HTPB [27].

(ii) The blocking effect on surface heat transfer depends only on the gas blowing rate. Referring only to the volatile fuel mass flow, the density $\rho_{v}=(1-$ k) $\rho_{F}$ should be used instead of $\rho_{F}$ in the previous equations (1) and (2). It follows that, disregarding other effects, the regression rate should be nearly inversely proportional to $(1-k)$.

(iii) The value of $\Delta H_{v \text {,eff }}$ (here evaluated on a volatile binder mass basis) is higher than the value of the volatile binder alone $\Delta H_{v}$, as further energy is needed to heat the nonvolatile particle to the surface temperature. Nevertheless, due to the relatively low heat capacity of most metal additives, $\Delta H_{v \text {, eff }}$ does not differ much from $\Delta H_{v}$. The new value of $\Delta H_{v \text {,eff }}$ should be used to evaluate $B$, but the resulting effect is mitigated by the low exponent of $B$ in (2). (Note that a different point of view is often adopted in the literature: reference is made to the whole mass of the fuel grain. In this case $\Delta H_{v \text {,eff }}$ is decreased as the nonvolatile particle should be only heated to surface temperature and not gasified.)

(iv) The radiant energy flux coming from condensed particles is large. This effect is mitigated by the coupling effect between radiant and convective heat fluxes.

(v) Additives enhance flame temperature and $\Delta H_{f w}$ increases. If particle size is large (some $\mu \mathrm{m}$ ) the nonvolatile particles perform distributed combustion releasing some heat far from the fuel surface. On the contrary, nanometric particles burn more rapidly and closer to the fuel surface. Also microexplosions may be experienced. Moreover particle coating affects combustion. The new value of $\Delta H_{f w}$ should be used to evaluate $B$, but the resulting effect is mitigated by the low exponent of $B$ in (2). (vi) The removal of solid from the grain surface may become the rate-limiting process for fuel rate regression [51]. The presence of flakes/agglomerates has been observed on the surface and in the combustion zone. Metals and their oxide may accumulate on the surface and leave it as agglomerates. At high mass fluxes the decomposition of fuel binders such as HTPB occurs very quickly and soot is formed, possibly due to desorption process of HTPB fragments at the grain surface.

(vii) Some of the oxidizer is consumed by metal particles burning and converted to solid oxides, thus reducing local gaseous mass-flux.

Beside metals, also oxidizer particles such as ammonium perchlorate (AP) have been used [52-55]. The presence of an oxidizer in the solid grain determines nearsurface/heterogeneous reactions. In addition the flame in the boundary layer approaches the surface, thus further enhancing heat transfer. Consequently, the regression rate increases. Nevertheless, the inherently safe inert behavior of the solid grain is compromised.

4.2. Energetic Compounds. Energetic formulations can be used in solid fuels to replace low-energy conventional binders such as HTPB. Both polymers and/or plasticizers which contribute to the overall energy of the composition can be used.

High nitrogen ingredients (HiN) show great promise [56, 57]. Despite low decomposition temperatures, energetic materials such as triaminoguanidinium azotetrazolate (TAGzT) present positive heat of formation. Containing no oxygen, the dominant chemistry is more centered on condensed phase reactions and much faster decomposition rates are obtained when compared to HMX. A 25\% increment in regression rate with respect to a similar HTPB grain has been observed using a $25 \% \mathrm{wt}$ addition of TAGzT [58]. If nanoaluminum is added in addition to HiN ingredients, $\mathrm{Al}$ reacts with the high-temperature nitrogen liberated from the decomposition of HiN material to form AlN near the surface where oxygen is absent.

Azido-substituted polymers such as glycidyl azide polymer (GAP) [59], 3,3-bis-(azidomethyl)oxetane (BAMO) and 3-azidomethyl-3-methyl oxetane (AMMO) are effective as the polymer matrix. However, these compounds begin to transition the inert fuel to a fuel-rich solid propellant and the safe nature of classical HREs may be compromised.

4.3. Paraffin Fuels. Researchers at Stanford University [60] discovered that paraffin-based fuels exist which have regression rates that are 3-4 times than those of conventional 
hybrid fuels. This is mainly due to the production of a thin liquid layer on the fuel grain surface which becomes instable: If this layer is characterized by low viscosity $\mu$ and low surface tension $\sigma$, instability driven by the oxidizer flow may arise and liquid fuel droplets are injected into the boundary layer $[5,61]$. This mass-transfer mechanism is similar to a spray injection and does not depend on heat transfer. It enhances fuel mass flow without the blocking effect typical of gaseous fuel blowing. The entrained mass flow $\dot{m}_{\text {entr }}$ depends on dynamic pressure $p_{\text {dyn }}$, layer thickness $h$, and liquid properties (i.e., $\mu$ and $\sigma$ ) at the temperature of the melt layer

$$
\dot{m}_{\mathrm{entr}} \propto \frac{p_{\mathrm{dyn}}^{\beta} h^{\delta}}{\sigma^{\theta} \mu^{\lambda}} .
$$

As shown in (9), entrainment occurs only when the liquid in the melt layer has low values of $\mu$ and $\sigma$. For example, high-density polyethylene (HDPE) does form a melt layer, but $\mu$ is four orders of magnitude larger than paraffin and droplet entrainment is not significant. In contrast, fuels such as paraffin waxes and polyethylene waxes have low viscosity. These fuels also present other advantages, as they are lowcost, easy to process, and environmentally benign. They may give a specific impulse similar to kerosene, but with higher density and without the combustion product toxicity typical of SRM grains. A blend of different components can be used to mitigate this issue and to tailor regression rate for a given mission. For example, ethylene-vinyl acetate copolymers have been mixed with paraffin-wax to change the melted-fuel viscosity [62]. According to experimental data, regression rate increases almost in proportion with $\mu^{-1 / 6}$. Beside all these advantages, one issue is the low mechanical strength. Additives may be used to improve this quality, but they may affect viscosity and surface tension adversely.

Additives to further enhance the regression rate have also been used. As an example high regression rate has been experienced adding $\mathrm{Al}$ particles in paraffin-based fuels: $13 \%$ wt of aluminum particles (Silberline) determine a 30\% regression rate enhancement [8]. It is believed that aluminum particles coming from the surface may be encapsulated by the wax, thus helping ignition. Regression rate usually increases with loaded weight of additive. This is not the case of some tests with ammonia borane [48], where regression rate was enhanced with small weight fraction, while decreased for higher weight fractions.

4.4. Cryogenic Fuels. Many fuels such as methane, ethylene, pentane, and RP1 have been tested as solid cryogenic grains [63-65]. They proved to regress 2 to 10 times faster than storable fuels in the same operating conditions. Solid methane/gaseous oxygen (GOX) proved to regress at nearly $8 \mathrm{~mm} / \mathrm{s}$ with an oxidizer mass flux of $20 \mathrm{~kg} / \mathrm{m}^{2} / \mathrm{s}$. Also, solid oxidizers such as $\mathrm{CO}$ and $\mathrm{O}_{2}$ have been tested (reverse HRE configuration) [66]. The high regression rate obtained is explained by the theories developed to describe high regression rates in paraffin fuels $[5,61]$. Results are encouraging, but complications arise due to the low temperatures, and the production of large grain is difficult.

\section{Nonconventional Grain Geometries and Injectors}

Methods can be used that modify classical flow structure in order to improve heat transfer. These approaches may be based on nonconventional grain geometry, nonconventional injector design, or both. Nonconventional geometries, for example, radial and/or end burning of the grain, may determine 3D flows with detachments and recirculation. Swirl injectors are able to reduce both thickness and growth of the boundary layer, thus enhancing heat transfer. The heat transfer variation is reduced and regression rate is more uniform.

5.1. Head-End Swirl in Side-Burning Grains. Several researchers tested swirl injector at the fore end of conventional cylindrical grains $[11,67]$. For instance, Yuasa et al. [11] reports results for different grain length, geometric swirl factor, and $G_{O}$. Swirl strength and oxidizer mass flux were varied independently. An axial injector was also used to obtain a baseline correlation. A regression rate 2.7 times greater than that for the baseline case was experienced using GOX and PMMA. Using the correlation $r=a G_{O}^{n}$ it has been found that the exponent $n$ is not affected significantly by the swirl number, but changes with the range of mass flux ( $n \sim 0.8$ for large values of $G_{O}$ according to the theoretical turbulent layer heat transfer, while $n \sim 0.6$ at lower $G_{O}$ values, probably due to radiation effects). On the contrary, the value of $a$ increases almost linearly with swirl factor.

Some researchers also modified the grain geometry [67, 68]. Lee et al. [67] considered grooves in the grain and swirl injectors. Separated and combined effects where analyzed. Swirl injectors alone are able to have double-regression rate, while grooves have a minor effect if used alone. Grooves are supposed to enhance the turbulence in the near-surface region and allow for an increased burning surface of more than $100 \%$, but the complexity and the cost associated with grain manufacture must be taken into account. In [68] a tapered grain is proposed to avoid swirl decay along the grain axis. Conservation of angular momentum accelerates the tangential velocity because of section reduction and the swirl angle may be maintained.

It is to be pointed out that some effects of swirling must be addressed, including torque and effects of nonaxial flow in the nozzle (effective throat area and divergence losses). This holds for any of the concepts employing swirl oxidizer injection.

\subsection{Aft-End Swirl in Side-Burning Grains: The Vortex Hybrid.} The key characteristic of vortex hybrid $[10,69,70]$ is a unique coaxial, coswirling, counterflowing vortex pair which has been found to improve regression rate. A classical cylindrical grain with circular port section is used. The vortex pair is obtained by injecting the oxidizer through a swirl injector located between the aft end of the fuel grain and the inlet of the exit nozzle. The oxidizer is prevented from directly flowing out of the nozzle by centrifugal forces and pressure gradients. An outer vortex is formed which spirals toward the engine head end. At the engine head end, the outer 
vortex turns inward and transforms into an inner vortex that spirals toward the nozzle. This flow structure presents many advantages: the residence time is increased, mixing is enhanced, both thickness and growth of the boundary layer are reduced, thus augmenting the heat transfer to the fuel surface and making the regression rate very uniform. Tests have been carried out using GOX with HTPB and other proprietary fuels. HTPB shows a regression rate up to 6 times faster that those in HREs with classical head end injectors. Using the classical correlation of (3), it has been found [14]:

$$
r=0.193 G_{O}^{0.54}
$$

The empirical power of 0.54 on mass flux, in conjunction with the single circular port and the uniform regression rate along the axis, guarantees a reduced mixture ratio shifting. On the other hand, the regression rate depends on the injection velocity of the oxidizer. Maximum mass flux tested [10] was about $100 \mathrm{~kg} / \mathrm{m}^{2}$ s. Scale effects must be investigated.

5.3. Radial-Flow HREs. An alternative, which may improve fuel section volumetric efficiency, is the radial flow hybrid rocket. Multiple fuel plates stacked on top of each other can provide very large burn surface areas and high volumetric efficiency if there is a little gap in between the plates. This configuration can be a good option when a small $L / D$ is important as in launcher upper stages. It can be also used for space propulsion [71]. The flow can be injected from a central hole in a disk, or radially. The measured regression rates are greater than those observed in axial port designs at the same flux and chamber pressure levels [12]. However, detailed regression on both upper and lower fuel disks is very complex, being influenced by flow impingement, flow separation, and 3D flows that cause nonuniform regression rate, determining the presence of sliver. In addition, structural integrity may be of concern if multiple disks are used.

Swirl have been used in radial flow hybrids. One example is the so called vortex flow pancake (VFP) [72] where the swirl injectors are between two end-burning disks of fuel. It has been found that the regression rate is highly dependent on flux. The regression rate appears to be one order of magnitude larger that the one obtained in classical HRE for similar mass fluxes. Nevertheless, the mass flux used for these tests are small with respect to typical values in classical HRE tests: Scale effects must be evaluated.

\subsection{Side/End-Burning Grains: The Cascaded Multistage} Impinging-Jet. The cascaded multistage impinging-jet (CAMUI) is a method proposed to improve thrust level of hybrid rockets without changing fuel material or introducing nonconventional injectors $[13,73]$. This concept is based on a unique geometry of the fuel grain which consists of several stages of cylindrical blocks with two axial ports. The jet of the combustion gas collides with the burning surfaces repeatedly, resulting in the high regression rate of the solid fuel. Both side burning and lateral end burning is used, thus reducing grain length for a given $A_{b}$. In fact, each block has three burning surfaces, namely, the port surfaces and the two lateral ends (upstream and downstream end faces). To reduce sliver and avoid grain collapsing, the ports must reach the external grain diameter when the upstream face meets the downstream end face. The three surfaces have different regression and predicting grain geometry evolution and fuel flow rate is not an easy task. The development of accurate regression rate correlation is needed. Tests have been performed using GOX/PE propellant combination. The regression rate for port surface is similar to (3):

$$
r=a\left(\alpha^{\prime}\right) G_{p}^{n}
$$

but in this case $a$ is a function of the local mixture ratio $\alpha^{\prime}$ and the propellant mass flux $G_{p}$ is used. The regression rate of forward end faces depends on the spacing between blocks $H$ and the port area diameter $D_{p}$ of the upstream block:

$$
\frac{r}{\left(H / D_{p}\right)^{m}}=a\left(\alpha^{\prime}\right) G_{p}^{n}
$$

The downstream end face has a more complicated behavior, presenting non uniform regression rates. Two different regression rate correlations are introduced as wall jets enhance the regression in the central part of the face. Due to the grain configuration low values of $L / D$ can be obtained, but volumetric efficiency and sliver may be issues.

\section{Final Remarks}

The low regression rate of fuel grain is one of the most challenging issues for hybrid rockets. Many solutions have been presented in the literature, demonstrating the vital research activity in hybrid rocket propulsion. The most important principles and investigated solutions have been reviewed here. The challenge is to eliminate or, at least, mitigate the negative effects of the low regression rate, without compromising the appealing features of HREs (e.g., safety, low cost, simplicity, environmental friendliness).

Propellant combinations which require high mixture ratios reduce the contribution grain fuels make. In this case the choice of the oxidizer plays a significant role. Proper tools which couple engine design and trajectory optimization are needed to find the best propellant combination to be used. In order to have acceptable rocket geometries, multiport grains have to be adopted for missions requiring high performance. However, the drawbacks of multiport grains, such as sliver and structural concerns, drive the researchers to investigate means which enhance the regression rate.

Approaches which increase regression rate have been presented in two sections of this paper. A first section has been devoted to advanced fuels, considering both additives (mainly metal particles) and nonconventional fuels, that is, paraffin-based fuels and cryogenic grains. A second section considered methods which aim at increasing the heat transfer to the grain surface, modifying grain and/or injector geometry. Figure 4 and Table 1 compare the performance of approaches reviewed here. Dashed lines shows conventional HRE regression rate for comparison. Higher regression rates are usually obtained with respect to classical HREs at similar operating conditions. Most of the solutions presented remain 
at a laboratory or concept scale and need further research efforts. Nevertheless, the data presented in the literature show that promising approaches do exist and may be adopted/combined to give HREs the opportunity to become a very competitive propulsion system.

\section{Nomenclature}

\begin{tabular}{|c|c|}
\hline$A_{b}:$ & Burning surface area, $\mathrm{m}^{2}$ \\
\hline$A_{p}:$ & Port area, $\mathrm{m}^{2}$ \\
\hline$A_{t}:$ & Nozzle throat area, $\mathrm{m}^{2}$ \\
\hline$a:$ & Regression constant, $\mathrm{m}^{1+2 n} \mathrm{~kg}^{-n} \mathrm{~s}^{n-1}$ \\
\hline$B:$ & Blowing parameter \\
\hline$D:$ & Rocket diameter, $\mathrm{m}$ \\
\hline$D_{g}:$ & Grain outer diameter, $\mathrm{m}$ \\
\hline$D_{h}^{\circ}:$ & Hydraulic diameter, $\mathrm{m}$ \\
\hline$D_{p}:$ & Port diameter, $\mathrm{m}$ \\
\hline$c:$ & Effective exhaust velocity, $\mathrm{m} / \mathrm{s}$ \\
\hline$c^{*}:$ & Characteristic velocity, $\mathrm{m} / \mathrm{s}$ \\
\hline$C_{F}:$ & Thrust coefficient \\
\hline$F:$ & Thrust magnitude, $\mathrm{N}$ \\
\hline$G$ : & Mass flux, $\mathrm{kg} / \mathrm{m}^{2}$ \\
\hline$k:$ & Additive loading, $\%$ wt \\
\hline$L:$ & Overall length, $\mathrm{m}$ \\
\hline$L_{g}:$ & Grain length, $\mathrm{m}$ \\
\hline$m:$ & Mass, kg \\
\hline$n:$ & Mass-flux exponent \\
\hline$p_{\text {dyn }}:$ & Dynamic pressure, $\mathrm{Pa}$ \\
\hline$P:$ & Burning perimeter, $\mathrm{m}$ \\
\hline$q:$ & Total heat flux, $\mathrm{W} / \mathrm{m}^{2}$ \\
\hline$q_{c}:$ & Convective heat flux, $\mathrm{W} / \mathrm{m}^{2}$ \\
\hline$q_{r}:$ & Radiant heat flux, W/m² \\
\hline$r:$ & Regression rate, $\mathrm{m} / \mathrm{s}$ \\
\hline $\operatorname{Re}_{D}:$ & Reynolds number \\
\hline$w:$ & Web thickness, $\mathrm{m}$ \\
\hline$x:$ & Axis abscissa, $\mathrm{m}$ \\
\hline$\alpha:$ & Mixture ratio \\
\hline$\Delta H_{f w}:$ & $\begin{array}{l}\text { Energy of the main stream relative to the } \\
\text { surface, } \mathrm{J} / \mathrm{kg}\end{array}$ \\
\hline$\Delta H_{v}:$ & Binder (volatile) gasification heat, J/kg \\
\hline$\Delta H_{v, \mathrm{eff}}:$ & Effective gasification heat, J/kg \\
\hline$\epsilon:$ & Nozzle area-ratio \\
\hline$\mu:$ & Dynamic viscosity, $\mathrm{Pa} s$ \\
\hline$\rho:$ & Density, $\mathrm{kg} / \mathrm{m}^{3}$ \\
\hline$\sigma:$ & Surface tension, $\mathrm{N} / \mathrm{m}$. \\
\hline
\end{tabular}

\section{Superscripts}

$\because$ Time derivative.

\section{Subscripts}

$\begin{array}{ll}c: & \text { Combustion chamber } \\ e: & \text { Nozzle exit } \\ \text { entr: } & \text { Entrained } \\ F: & \text { Fuel } \\ i: & \text { Initial } \\ O: & \text { Oxidizer } \\ p: & \text { Overall propellant (oxidizer }+ \text { fuel). }\end{array}$

\section{References}

[1] M. A. Dornheim, "Reaching 100 km," Aviation Week and Space Technology, vol. 161, no. 6, pp. 45-46, 2004.

[2] J. Benson, "Safe and affordable human access to LEO," in Proceedings of the AIAA Space Conference \& Exhibition, pp. 1215-1216, September 2005, AIAA Paper 2005-6758.

[3] S. Gordon and B. J. McBride, "Computer program for calculation of complex chemical equilibrium compositions and applications," NASA Reference Publication 1311, 1996.

[4] G. Sutton, Rocket Propulsion Elements: An Introduction to the Engineering of Rockets, Wiley, NewYork, NY, USA, 6th edition, 1992.

[5] M. A. Karabeyoglu, D. Altman, and B. J. Cantwell, "Combustion of liquefying hybrid propellants: part 1, General theory," Journal of Propulsion and Power, vol. 18, no. 3, pp. 610-620, 2002.

[6] G. A. Risha, B. J. Evans, E. Boyer, and K. K. Kuo, "Metals, energetic additives, and special binders used in solid fuels for hybrid rockets," in Fundamentals of Hybrid Rocket Combustion and Propulsion, M. J. Chiaverin and K. K. Kuo, Eds., vol. 218 of Progress in Astronautics and Aeronautics, chapter 10, pp. 413456, AIAA, Reston, Va, USA, 2007.

[7] D. J. Gramer and E. E. Rice, "Experimental investigation of a metallized cryogenic hybrid rocket engine," in Proceedings of the 34th AIAA/SAE/ASME/ASEE Joint Propulsion. Conference and Exhibit, July 1998, AIAA Paper 98-3509.

[8] B. Evans, E. Boyer, K. K. Kuo, G. Risha, and M. Chiaverini, "Hybrid rocket investigations at penn state university's high pressure combustion laboratory: overview and recent results," in Proceedings of the 45th AIAA/ASME/SAE/ASEE Joint Propulsion Conference and Exhibit, August 2009.

[9] P. George, S. Krishnan, P. M. Varkey, M. Ravindran, and L. Ramachandran, "Fuel regression rate in hydroxyl-terminatedpolybutadiene/gaseous-oxygen hybrid rocket motors," Journal of Propulsion and Power, vol. 17, no. 1, pp. 35-42, 2001.

[10] W. H. Knuth, M. J. Chiaverini, J. A. Sauer, and D. J. Gramer, "Solid-fuel regression rate behavior of vortex hybrid rocket engines," Journal of Propulsion and Power, vol. 18, no. 3, pp. 600-609, 2002.

[11] S. Yuasa, K. Yamamoto, H. Hachiya, K. Kitagawa, and Y. Oowada, "Development of a small sounding hybrid rocket with a swirling-oxidizer-type engine," in Proceedings of the 37th AIAA/ASME/SAE/ASEE Joint Propulsion Conference, 2001, AIAA Paper 2001-3537.

[12] J. R. Caravella Jr., S. D. Heister, and E. J. Wernimont, "Characterization of fuel regression in a radial flow hybrid rocket," Journal of Propulsion and Power, vol. 14, no. 1, pp. 5156, 1998.

[13] H. Nagata, S. Hagiwara, T. Totani, and T. Uematsu, "Optimal fuel grain design method for CAMUI type hybrid rocket," in Proceedings of the 47th. AIAA/ASME/SAE/ASEE Joint Propulsion Conference and Exhibit, 2011, AIAA Paper 2011-6105.

[14] M. J. Chiaverini, "Review of solid-fuel regression rate behavior in classical and nonclassical hybrid rocket motors," in Fundamentals of Hybrid Rocket Combustion and Propulsion, M. J. Chiaverin and K. K. Kuo, Eds., vol. 218 of Progress in Astronautics and Aeronautics, chapter 2, pp. 37-125, AIAA, Reston, Va, USA, 2007.

[15] G. A. Marxman and M. Gilbert, "Turbulent boundary layer combustion in the hybrid rocket," in Proceedings of the 9th International Symposium on Combustion, pp. 371-383, Academic Press, 1963. 
[16] G. A. Marxman, C. E. Wooldridge, and R. J. Muzzy, "Fundamentals of hybrid boundary layer combustion," in Heterogeneous Combustion, H. G. Wolfhard, I. Glassman, and L. Green Jr., Eds., vol. 15 of Progress in Astronautics and Aeronautics, pp. 485-521, Academic Press, New York, NY, USA, 1964.

[17] G. A. Marxman, "Combustion in the turbulent boundary layer on a vaporizing surface," in Proceedings of the 10th International Symposium on Combustion, pp. 1337-1349, Combustion Institute, 1965.

[18] R. J. Muzzy, "Applied hybrid combustion theory," in Proceedings of the 8th Joint Propulsion Specialist Conference (AIAA/SAE '72), 1972, AIAA Paper No. 72-1143.

[19] D. Altman and R. Humble, "Hybrid rocket propulsion systems," in Space Propulsion Analysis and Design, R. W. Humble, G. N. Henry, and W. J. Larson, Eds., p. 379, The Mc Graw-Hill Companies, Primis Custom, New York, NY, USA, 1995.

[20] P. J. Paul, H. S. Mukunda, and V. K. Jain, "Regression rates in boundary layer combustion," in Proceedings of the 19th International Symposium on Combustion, pp. 717-729, Combustion Institute, 1982.

[21] P. N. Estey, D. Altman, and J. S. McFarlane, "An evaluation of scaling effects for hybrid rocket motors," in Proceedings of the 27th Joint Propulsion Conference (AIAA/SAE/ASME '91), Sacramento, Calif, USA, June 1991, AIAA Paper 91-2517.

[22] L. D. Strand, M. D. Jones, R. L. Ray, and N. S. Cohen, "Characterization of hybrid rocket internal heat flux and HTPB fuel pyrolysis," in Proceedings of the 30th Joint Propulsion Conference, June 1994, AIAA Paper 94-2876.

[23] L. D. Smoot and C. F. Price, "Pressure dependence of hybrid fuel regression rates," AIAA Journal, vol. 5, no. 1, pp. 102-106, 1966.

[24] F. J. Kosdon and F. A. Williams, "Pressure dependence of nonmetalized hybrid fuel regression rate," AIAA Journal, vol. 5, no. 4, pp. 774-777, 1967.

[25] R. N. Kumar and D. B. Stickler, "Polymer-degradation theory of pressure-sensitive hybrid combustion," in Proceedings of the 3th International Symposium on Combustion, pp. 1059-1072, Combustion Institute, 1971.

[26] E. Miller, "Hybrid rocket combustion regression rate model," AIAA Journal, vol. 4, no. 4, pp. 752-753, 1966.

[27] M. J. Chiaverini, N. Serin, D. K. Johnson, Y. C. Lu, K. K. Kuo, and G. A. Risha, "Regression rate behavior of hybrid rocket solid fuels," Journal of Propulsion and Power, vol. 16, no. 1, pp. 125-132, 2000.

[28] M. J. Chiaverini, K. K. Kuo, A. Peretz, and G. C. Harting, "Regression-rate and heat-transfer correlations for hybrid rocket combustion," Journal of Propulsion and Power, vol. 17, no. 1, pp. 99-110, 2001.

[29] W. M. Kays and M. E. Crawford, Convective Heat and Mass Transfer, McGrawHill, New York, NY, USA, 2nd edition, 1980.

[30] D. A. Kearney, K. F. Joiner, M. P. Gnau, and M. A. Casemore, "Improvements to the marketability of hybrid propulsion technologies," in Proceedings of the AIAA Space Conference, pp. 1674-1688, September 2007, AIAA Paper 2007-6144.

[31] L. Casalino and D. Pastrone, "Optimal design of hybrid rocket motors for microgravity platform," Journal of Propulsion and Power, vol. 24, no. 3, pp. 491-498, 2008.

[32] A. Ben-Yakar and A. Gany, "Hybrid engine design and analysis," in Proceedings of the 29th Joint Propulsion Conference, June 1993, AIAA Paper 93-2548 .

[33] L. Casalino, D. Pastrone, and F. Simeoni, "Hybrid rocket upper stage optimization: efects of grain geometry," in Proceedings of the 47th AIAA/ASME/SAE/ASEE Joint Propulsion Conference and Exhibit, 2011, AIAA Paper 2011-6024.

[34] J. Fuller, P. Lu, R. Jansen, and J. Hoffman, "Advantages of rapid prototyping for hybrid rocket motor fuel grain fabrication," in Proceedings of the 47th AIAA/ASME/SAE/ASEE Joint Propulsion Conference and Exhibit, 2011, AIAA Paper 2011-5821.

[35] L. Casalino and D. Pastrone, "Optimal design and control of hybrid rockets for access to space," in Proceedings of the 41st AIAA/ASME/SAE/ASEE Joint Propulsion Conference and Exhibit, July 2005.

[36] L. Casalino and D. Pastrone, "Optimization of hybrid sounding rockets for hypersonic testing," in Proceedings of the 45th AIAA/ASME/SAE/ASEE Joint Propulsion Conference and Exhibit, August 2009.

[37] L. Casalino and D. Pastrone, "Optimal design of hybrid rocket motors for launchers upper stages," Journal of Propulsion and Power, vol. 26, no. 3, pp. 421-427, 2010.

[38] E. J. Wernimont and S. D. Heister, "Combustion experiments in hydrogen peroxide/polyethylene hybrid rocket with catalytic ignition," Journal of Propulsion and Power, vol. 16, no. 2, pp. 218-326, 2000.

[39] M. Rocker, "Modeling of Nonacoustic Combustion Instability in Simulations of Hybrid Motor Tests," NASA/TP-000-209905, 2000.

[40] E. Doran, J. Dyer, K. Lohner, Z. Dunn, B. Cantwell, and G. Zilliac, "Nitrous oxide hybrid rocket motor fuel regression rate characterization," in Proceedings of the 43rd AIAA/ASME/ SAE/ASEE Joint Propulsion Conference, pp. 3485-3496, July 2007, AIAA Paper 2007-5352.

[41] L. D. Smoot and C. F. Price, "Regression rates of metalized hybrid fuel systems,” AIAA Journal, vol. 4, no. 5, pp. 910-915, 1965.

[42] L. D. Strand, R. L. Ray, and N. S. Cohen, "Hybrid rocket combustion study," in Proceedings of the 29th AIAA/ASME/ SAE/ASEE Joint Propulsion Conference and Exhibit, San Diego, Calif, USA, 1993, AIAA Paper 93-2412.

[43] H. R. Lips, "Heterogeneous combustion of highly aluminized hybrid fuels," AIAA Journal, vol. 15, no. 6, pp. 777-778, 1977.

[44] G. A. Risha, E. Boyer, R. B. WeHREan, and K. K. Kuo, "Performance comparison of HTPB-based solid fuels containing nano-sized energetic powder in a cylindrical hybrid rocket motor," in Proceedings of the 38th AIAA/ASME/SAE/ASEE Joint Propulsion Conference, 2002, AIAA Paper 2002-3576.

[45] E. Farbar, J. Louwers, and T. Kaya, "Investigation of metallized and nonmetallized hydroxyl terminated polybutadiene/hydrogen peroxide hybrid rockets," Journal of Propulsion and Power, vol. 23, no. 2, pp. 476-486, 2007.

[46] A. McCormick, E. Hultgren, M. Lichtman, J. Smith, R. Sneed, and S. Azimi, "Design, optimization, and launch of a $3^{\prime \prime}$ diameter $\mathrm{N}_{2} \mathrm{O}$ /aluminized paraffin rocket," in Proceedings of the 41st AIAA/ASME/SAE/ASEE Joint Propulsion Conference and Exhibit, 2005, AIAA Paper 2005-4095.

[47] B. Evans, A. Favorito, and K. Kuo, "Study of solid fuel burning-rate enhancement behavior in an X-ray translucent hybrid rocket motor," in Proceedings of the 41stAIAA/ASME/SAE/ASEE Joint Propulsion Conference and Exhibit, Tucson, Arizona, 2005, AIAA Paper 2005-3909.

[48] M. R. Weismiller, T. L. Connell Jr., G. A. Risha, and R. A. Yetter, "Characterization of ammonia borane (NH3BH3) enhancement to a paraffin fueled hybrid rocket system," in Proceedings of the 46th AIAA/ASME/SAE/ASEE Joint Propulsion Conference and Exhibit, 2010, AIAA Paper 2010-6639. 
[49] L. Galfetti, L. Merotto, M. Boiocchi, F. Maggi, and L. De Luca, "Ballistic and rheological characterization of paraffinbased fuels for hybrid rocket propulsion," in Proceedings of the 47th AIAA/ASME/SAE/ASEE Joint Propulsion Conference and Exhibit, 2011, AIAA Paper 2011-5680.

[50] D. B. Larson, E. Boyer, T. Wachs et al., "Characterization of the performance of paraffin / LiAlH4 solid fuels in a hybrid rocket system," in Proceedings of the 47th AIAA/ASME/SAE/ASEE Joint Propulsion Conference and Exhibit, San Diego, Calif, USA, 2011, AIAA Paper 2011-5822.

[51] L. D. Strand, R. L. Ray, F. A. Anderson, and N. S. Cohen, "Hybrid rocket fuel combustion and regression rate study," in Proceedings of the AIAA, SAE, ASME, and ASEE, Joint Propulsion Conference and Exhibit, July 1992, AIAA Paper 1992-3302.

[52] B. N. Raghunandan, E. R. Ravichandran, and A. G. Marathe, "Combustion related to solid-fuel ramjets," Journal of Propulsion and Power, vol. 1, no. 6, pp. 502-504, 1985.

[53] P. George, S. Krishnan, P. M. Varkey, M. Ravindran, and L. Ramachandran, "Fuel regression rate in hydroxyl-terminatedpolybutadiene/gaseous-oxygen hybrid rocket motors," Journal of Propulsion and Power, vol. 17, no. 1, pp. 35-42, 2001.

[54] L. R. Knox, M. D. Moser, and R. A. Frederick Jr., "Mixed hybrid propellants," in Proceedings of the 39th AIAA/ASME/SAE/ASEE Joint Propulsion Conference and Exhibit, 2003, AIAA Paper 2003-4745.

[55] R. A. Frederick Jr., J. J. Whitehead, L. R. Knox, and M. D. Moser, "Regression rates study of mixed hybrid propellants," Journal of Propulsion and Power, vol. 23, no. 1, pp. 175-180, 2007.

[56] D. E. Chavez and M. A. Hiskey, "1,2,4,5-tetrazine based energetic materials," Journal of Energetic Materials, vol. 17, no. 4, pp. 357-377, 1999.

[57] S. F. Son, H. L. Berghout, C. A. Bolme, D. E. Chavez, D. Naud, and M. A. Hiskey, "Burn rate measurements of HMX, TATB, DHT, DAAF, and BTATz," in Proceedings of the Combustion Institute, vol. 28, pp. 919-924, 2000.

[58] A. Risha, A. G. Miller, R. A. Glass, V. A. Yeager, M. J. Chiaverni, and B. C. Tappan, "Regression rates of solid fuels containing high nitrogen (HiN) materials," in Proceedings of the 47th AIAA/ASME/SAE/ASEE Joint Propulsion Conference and Exhibit, August 2011, AIAA Paper 2011-5912.

[59] K. Hori et al., "Combustion characteristics of hybrid rocket motor using GAP as a solid fuel (II)," in Proceedings of the 47th AIAA/ASME/SAE/ASEE Joint Propulsion Conference and Exhibit, 2011, AIAA Paper 2011-5819.

[60] M. A. Karabeyoglu, B. J. Cantwell, and D. Altman, "Development and testing of paraffin-based hybrid rocket fuels," in Proceedings of the 37th AIAA/ASME/SAE/ASEE Joint Propulsion Conference and Exhibit, July 2001, AIAA Paper 2001-4503.

[61] M. A. Karabeyoglu and B. J. Cantwell, "Combustion of liquefying hybrid propellants: part 2, Stability of liquid films," Journal of Propulsion and Power, vol. 18, no. 3, pp. 621-630, 2002.

[62] I. Nakagawa and S. Nikone, "Study on the regression rate of paraffin-based hybrid rcket fuel," Journal of Propulsion and Power, vol. 27, no. 6, pp. 1276-1279, 2011.

[63] P. G. Carrick and W. C. Larson, "Lab scale test and evaluation of cryogenic solid hybrid rocket fuels," in Proceedings of the 31st AIAA/ASME/SAE/ASEE Joint Propulsion Conference and Exhibit, 1995, AIAA Paper 95-2948.
[64] M. E. De Rose, K. L. Pfeil, P. G. Carrick, and W. C. Larson, "Tube burner studies of cryogenic solid combustion," in Proceedings of the 33rd AIAA/ASME/SAE/ASEE Joint Propulsion Conference and Exhibit, 1997, AIAA Paper 97-3076.

[65] E. E. Rice, M. J. Chiaverini, C. P. St.Clair, W. H. Knuth, and R. J. Gustafson, "Mars ISRU CO/O $\mathrm{O}_{2}$ hybrid engine development status," in Proceedings of the 38th Aerospace Sciences Meeting, 2000, AIAA Paper 2000-1066.

[66] C. P. St.Clair, E. E. Rice, W. H. Knuth, and D. J. Gramer, "Advanced cryogenic solid hybrid rocket engine developments: concept and testing," in Proceedings of the 34th AIAA/ASME/SAE/ASEE Joint Propulsion Conference and Exhibit, 1998, AIAA Paper 98-3508.

[67] C. Lee, Y. Na, and G. Lee, "The enhancement of regression rate of hybrid rocket fuel by helical grain configuration and swirl flow," in Proceedings of the AIAA/ASME/SAE/ASEE Joint Propulsion Conference and Exhibit, 2005, AIAA Paper 20053906.

[68] R. Wilkinson, K. Hart, R. Day, and I. Coxhill, "Proof-ofconcept testing of a sustained vortex-flow configuration for hybrid rocket motors," in Proceedings of the 46th AIAA/ASME/ SAE/ASEE Joint Propulsion Conference and Exhibit, July 2010, AIAA Paper 2010-6782.

[69] W. H. Knuth, M. J. Chiaverini, D. J. Gramer, and J. A. Sauer, "Numerical simulation of vortex-driven, hybrid rocket engines," in Proceedings of the 34th AIAA/ASME/SAE/ASEE Joint Propulsion Conference and Exhibit, 1998, AIAA Paper 983351.

[70] J. Madjalani, "Vortex injection hybrid," in Fundamentals of Hybrid Rocket Combustion and Propulsion, M. J. Chiaverin and K. K. Kuo, Eds., vol. 218 of Progress in Astronautics and Aeronautics, chapter 6, pp. 247-276, AIAA, Reston, Va, USA, 2007.

[71] R. Jansen and E. Teegarden, "Characterization of a vortexflow end-burning hybrid rocket motor for nanosatellite applications," in Proceedings of the AIAA/ASME/SAE/ASEE Joint Propulsion Conference and Exhibit, 2012, AIAA Paper 20120125.

[72] G. Haag, M. Sweeting, and G. Richardson, "An alternative geometry hybrid rocket for spacecraft orbit transfer manoeuvers," in Proceedings of the International Astronautical Federation (IAF '00), 2000, IAF Paper 00-W.2.07.

[73] H. Nagata, "New fuel configurations for advanced hybrid rockets," in Proceedings of the International Astronautical Federation (IAF '98), Paris, France, 1998, IAF-98-S.3.09. 

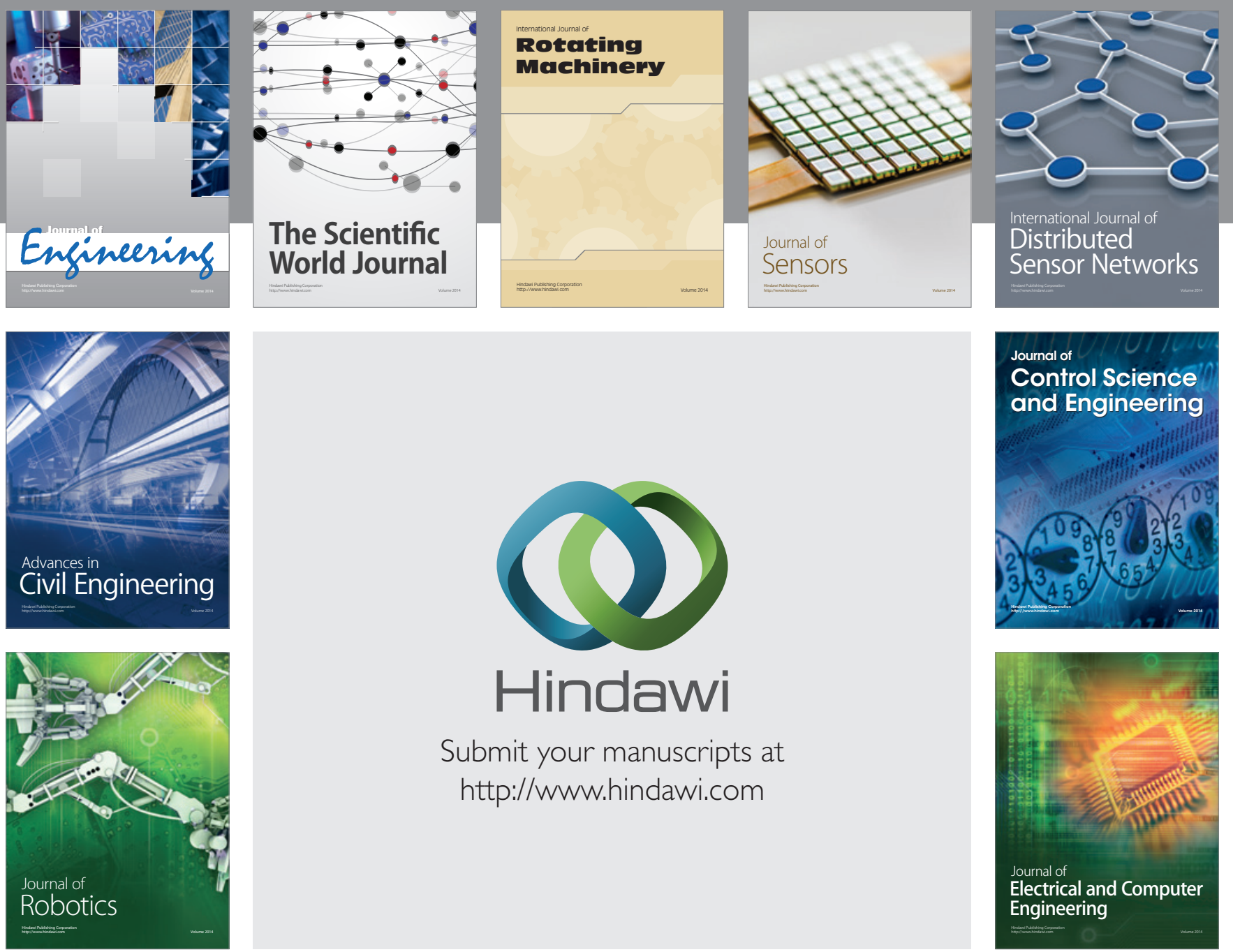

Submit your manuscripts at

http://www.hindawi.com
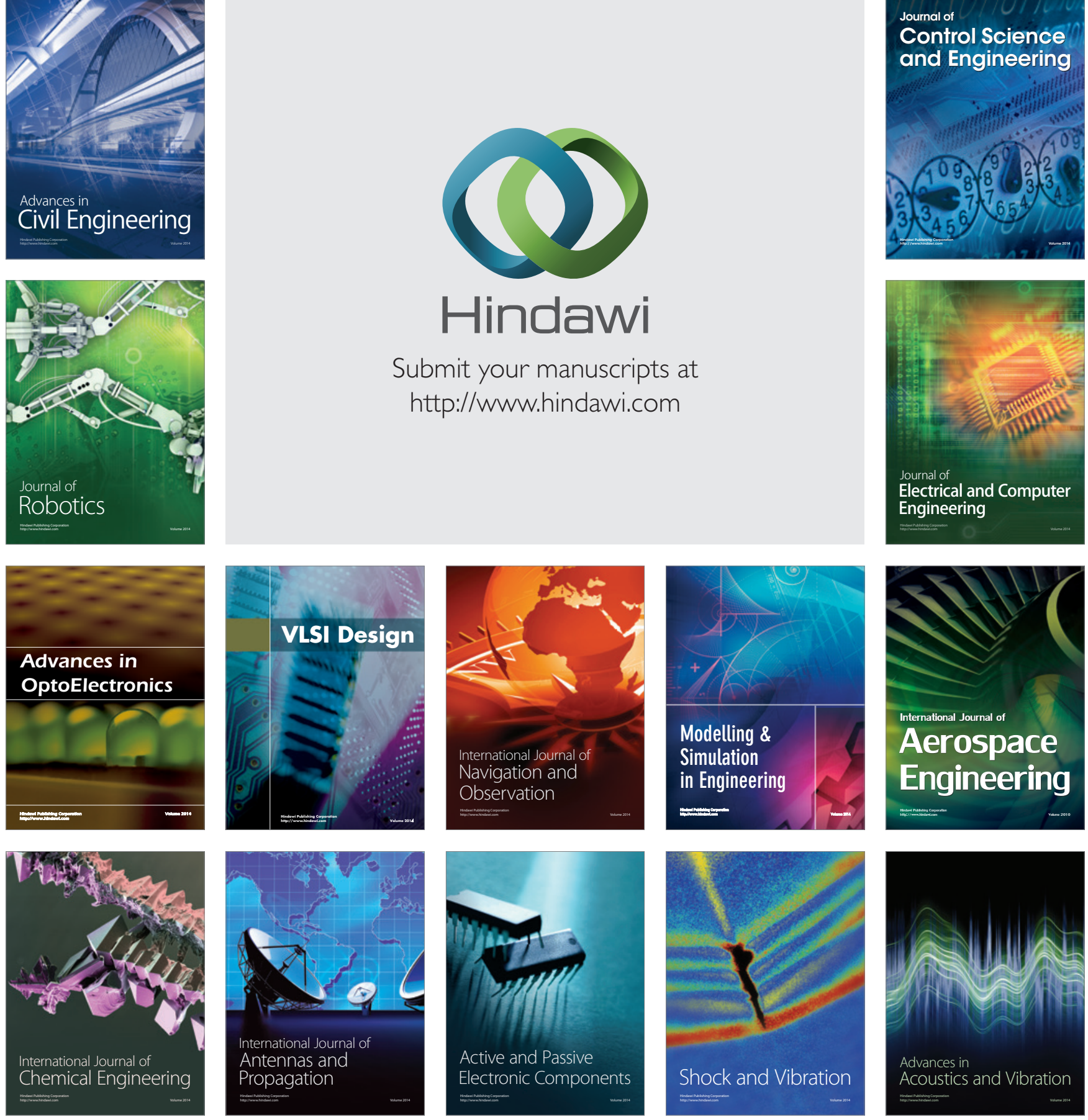